\title{
Is There a Need of Harmonization of Postgraduate Dermatology Curriculum in Nepal?
}

\author{
Agrawal S \\ Department of Dermatology \& Venereology, B. P. Koirala Institute of Health Sciences, Dharan, Nepal
}

P ostgraduate Dermatology Curricula aim to improve the quality of dermatological care in the country by producing competent dermatologists. The objectives of the program is to train a medical graduate who, at the end of training should be able to: diagnose and manage various diseases pertaining to the specialty of Dermatology, Venereology and Leprosy efficiently and effectively, backed by scientific knowledge and skills; develop empathetic and caring attitude and maintain high ethical standards practice; continue to evince keen interest in continuing education in the specialty irrespective of whether he/she is in a teaching institution or is a practicing specialist; be a motivated 'teacher'-defined as a specialist keen to share his/her knowledge and skills about dermatology, venereology and leprosy with a colleague or a junior or any learner (nurses and technician) and investigate and undertake research in a scientific manner to improve upon his/ her proficiency and that of the specialty in general.

\section{Post graduate Dermatology program in Nepal}

There are 20 medical colleges in Nepal spread over various parts of the country to take care of 28.5 million people (estimated in 2009). Most of the medical schools in the nation opened in the last 20 years. Most of which are in the private sector, though there are 4 government medical institutions as well. Post graduate teaching program exists in four universities officially and curricula are prepared by the academic council established by the university and it is being regulated by Nepal Medical Council. It was first started at B.P. Koirala Institute of Health Sciences (BPKIHS), Dharan in 2000AD. After that Institute of Medicine (IOM) started in 2004, National Academics of Medical Sciences (NAMS) in 2008 and then in other 9 medical colleges. On an average 5-8 dermatologists are being produced every year.

As an enrollment criteria, candidates must hold the MBBS or an equivalent degree recognized by the medical council of the country after successfully completing the qualifying examination. The selection is based on the merit obtained at the qualifying examination. The candidates may require service experience after completion of the internship however it is not mandatory and is varied from none to 1 year in different universities. A candidate pursuing the course should work in the institution as a full time student. No candidate should be permitted to run a clinic/laboratory/nursing home etc while studying postgraduate course. Each year should be taken as a unit for the purpose of calculating attendance and are required more than $80 \%$ of the attendance as an eligibility criteria for appearing in the final summative assessment.

The minimum length of training in dermatovenereology is 3 years. Learning in postgraduate program is essentially self-directed and primarily emanating from clinical and academic work. A list of teaching and learning activities are designed to facilitate students to acquire essential knowledge and skills. Although, a list of different topics of the subject are mentioned in most of the curricula, there is no emphasis on the specific learning objectives of different topics, no mention or no implementation of the must know, good to know or nice to know areas.

Lectures are kept to a minimum but certain topics are recommended common for post graduate students of all specialties eg bio-statistics, use of library, research methods, medical code of conduct and medical ethics, national Health and disease control programs, communication skills etc. These topics may preferably be taken up in the first few weeks of the $1^{\text {st }}$ year.

Journal club and subject seminar are recommended to be held once a week. All the postgraduate students are expected to attend and actively participate in discussion and enter it in the logbook with relevant details. Every candidate must make a presentation from the allotted journal(s), selected articles however there is no specified minimum number of presentation and no checklist for assessment of the presentation and participation. 
The postgraduate students must be posted in the ward, dermatology OPD, morning STD and HIV clinic and learning in field on rotations. They must attend weekly specialty clinics however in a few institutions limited facilities are available in the form of laboratories and specialties clinics.

Postings in other speciality/subspeciality and community also varies from institution to institution. Anandaban hospital is a leprosy hospital that provides experimental leprosy training. For this reason all universities send their residents to Anandaban hospital but the duration of posting is variable from 2 weeks to 6 weeks. Medicine posting may be required during dermatology residency however it varies from no to 6 months in different universities. Posting in other disciplines such as microbiology, pathology and plastic surgery are also not provided in all universities. As per Ministry of health of Nepal, 3 months community posting is compulsory however the universities opted as per their requirement either 3 months or changed to 3 hours per week.

Post graduate students must acquire teaching skills by teaching under graduate students (eg. medical, nursing) by taking demonstrations, bed side clinics, tutorials, lectures etc but is not being followed properly.

Every candidate pursuing degree course is required to carry out work on a selected research project under the guidance of a recognized post graduate teacher. The aim is to train a post graduate student in research methods and techniques. Every candidate must submit a research protocol of proposed thesis work within 6-9 months from the date of commencement of the course, on or before the dates notified by the university. Then the research protocol is reviewed and approved by the protocol and ethical committee of the University. No change in the thesis topic or guide is made without prior approval of the university. Thesis is certified by the guide, head of the department and head of the Institution and is submitted to the Dean (evaluation) at the end of second year or six months before final examination on or before the dates notified by the university. Thesis is valued by examiners appointed by the university and approval of thesis work is an essential precondition for a candidate to appear in the university examination. Although research is an important component for post graduate curriculum, the facilities in teaching departments are still meager in the developing countries and only a very few institutions can claim of good research facilities in dermatology for first-rate work.
Monitoring learning progress is essential to monitor the learning progress of each candidate through continuous appraisal and regular assessment. It helps not only teachers to evaluate students but also students to evaluate themselves. The monitoring is to be done by the staff of the department based on participation of students in various teaching / learning activities. Candidate is assessed using both workplacebased assessments and examination of knowledge and skills. Every candidate should maintain a work diary and record his/her participation in the training program conducted by the department such as journal reviews, seminars, etc. Special mention may be made of the presentations by the candidate as well as details of clinical or laboratory procedures, if any conducted by the candidate and signed by supervisor in a logbook.

Assessment tools are both formative and summative. Internal assessment (IA) as summative assessment varies in number from $0-3$ in different institutions. It may be only theory or both theory and practical. IA has weightage varying from $15-20 \%$. BPKIHS and NAMS give weightage to logbook also. Eligibility criteria for final evaluation include $80 \%$ attendance, satisfactory thesis with or without achievement of minimum number of procedures and presentation over three years. The resident needs to conclude the training with both practical and theory examinations. The number of theory paper varies from 3-4 papers in different institutions. The types of questions are multiple choice questions (MCQ), short answer question (SAQ), modified essay question (MEQ) or/and long answer question (LAQ). Similarly practical examination includes long case, semi-long case, short case, spotters, objective structured clinical examination (OSCE) and/ or viva and the clinical skills are tested in the practical examination. The length of the examination is $10-16$ hours for each candidate and the examination is completed in 2-3 days.

There are different assessment system for both formative and summative in different institutions. The assessments of candidates are done on diseases difficult to diagnose and untreatable cases or the emphasis is given by the examiners on theoretical aspects rather than practical day-to-day useful concepts of common diseases. Moreover, there is poor record in the logbook or the logbook is not implementing properly.

Finally, there is lack of uniform nomenclature of the specialty and its qualifications awarded by various universities in different institutes/ colleges within the country. The Post graduate degree in dermatology is awarded as MD (Dermatology \& Venereology), MD 
(Dermatology, \& VD) or MD (Dermatology \& STIs).

In conclusion, there is no uniformity in entry criteria for enrollment, lack of emphasis on specific learning objectives in curriculum, different assessment system, recruitment for teaching faculties and resources. Research facilities in postgraduate teaching departments are still inadequate. Furthermore, the facilities in the form of outdoor and indoor services, laboratories, accommodation, and ancillary staff are too meager in some institutions.

\section{Solution}

Though, Nepal medical council has implemented a licensing examination to overcome these challenges in postgraduate programs by assessing postgraduate competency prior to entering the profession. There is a great need for uniformity in postgraduate courses in dermatology to improve the quality of patient care in the era of globalization. It is desirable to have uniform standard curriculum with uniform nomenclature for postgraduate qualifications (degree and diploma) in the specialty awarded by various universities across the country. In addition, research facilities and evidence based dermatology practice should also be highlighted. A needs assessment can help to identify the discrepancies between current and ideal approaches in the postgraduate dermatology curriculum in revealing the present state of the curriculum and stakeholders priorities in those areas.

We all should try our best to improve and harmonize the dermatology curriculum to provide quality post graduate dermatology education and thus quality of dermatological care in the country. 